\title{
PREVALENCE OF HYPOTHYROIDISM IN A LARGE SERIES OF ADULT THALASSEMIC PATIENTS
}

${ }^{1}$ Radin R., ${ }^{1}$ Danesi L., ${ }^{1}$ Ambrogio A., ${ }^{2}$ Cassinerio E., ${ }^{2 Z}$ Zanaboni L., ${ }^{2}$ M. Baldini, ${ }^{2}$ Cappellini M.D., ${ }^{3}$ Poggi M., ${ }^{3}$ Ottaviani C., ${ }^{3}$ Toscano V., ${ }^{4}$ Zavattaro M., ${ }^{5}$ Cavagnini F., 1,2Persani L., ${ }^{2,6}$ Scacchi M.

${ }^{1}$ Istituto Auxologico Italiano, IRCCS San Luca, Division of Endocrinology and Metabolic Diseases, Milan, Italy; ${ }^{2}$ Department of Clinical Sciences and Community Health, University of Milan, Italy; ${ }^{3}$ Chair of Endocrinology, 2nd Faculty of Medicine, University of Rome La Sapienza, Italy; ${ }^{4}$ Department of Translational Medicine, Università del Piemonte Orientale "A. Avogadro", Novara, Italy ; 5 Istituto Auxologico Italiano, Laboratory of Neuroandocrine Research, Milan, Italy; "Istituto Auxologico Italiano, IRCCS San Giuseppe, Division of Endocrinology and Metabolic Diseases, Oggebbio-Piancavallo (VB), Italy

\section{INTRODUCTION}

Hypothyroidism has been traditionally described as a frequent endocrine complication of thalassemic children: in these patients hypothyroidism is mainly of primary origin and not linked to autoimmunity. Fewer data are available on the prevalence and nature of this complication in adult age thalassemia*. Therefore, we elected to evaluate thyroid function and autoimmunity in a large series of adult patients.

\section{STUDY DESIGN}

Ninety-five adult thalassemic subjects (48 women and 47 men, mean age $38.1 \pm 6.9$ years) underwent assessment of thyroid function (fT3, fT4 and TSH) and thyroid autoimmunity (anti-thyroid peroxidase and anti-thyroglobulin antibodies). Serum ferritin was also measured in all patients, who were regularly taking iron-chelating drugs. Hormone deficiencies other than hypothyroidism were adequately replaced.

\section{RESULTS}

Thirty-one patients (32.6\%), 16 men and 15 women, aged $39.5 \pm 6.7$ years, turned out to be affected by hypothyroidism. Two of them had previously undergone thyroid surgical removal for multinodular goiter and carcinoma, respectively. The stratification by age of the other hypothyroid subjects disclosed a prevalence of $25 \%(2 / 8)$ among those aged $20-30,27 \%$ (10/37) among those aged 30-40, and 36\% (16/44) among those aged 40-50. No hypothyroid patients displayed anti-TPO or anti-TG antibodies. No cases of secondary hypothyroidism were observed. Mean serum ferritin was not significantly different between hypothyroid and euthyroid thalassemic subjects $(1067.7 \pm 664.5 \mathrm{vs} 868.0 \pm 743.5 \mathrm{ng} / \mathrm{ml}$, NS, respectively).
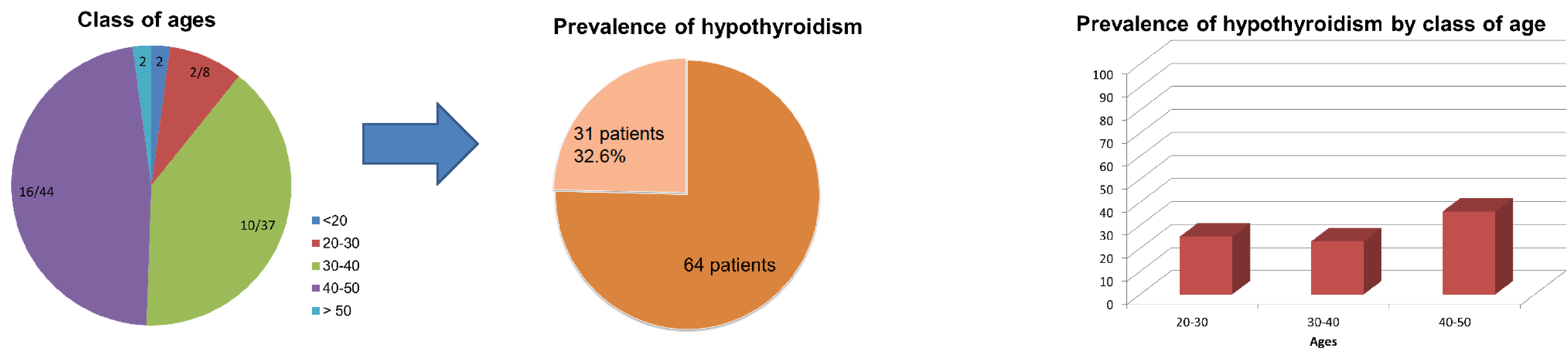

\section{CONCLUSIONS}

Hypothyroidism, primary in all cases, was highly prevalent in our series, being present in one third of the patients. This endocrine complication was equally distributed among genders and its prevalence appeared to progressively increase with age. Since measurement of serum ferritin expresses an estimation of the current degree of iron overload and not its evolution within the clinical history of patients, our data cannot rule out a contribution of secondary hemosiderosis to the pathogenesis of hypothyroidism in thalassemia. 